\title{
On Orthogonality Relations for Dual Discrete $q$-Ultraspherical Polynomials
}

\author{
Valentyna A. GROZA ${ }^{\dagger}$ and Ivan I. KACHURYK ${ }^{\ddagger}$ \\ $\dagger$ National Aviation University, 1 Komarov Ave., Kyiv, 03058 Ukraine \\ E-mail: groza@i.com.ua \\ $\ddagger$ Khmel'nyts'kyi National University, Khmel'nyts'kyi, Ukraine \\ E-mail: kachuryk@ief.tup.km.ua
}

Received February 14, 2006, in final form February 28, 2006; Published online March 16, 2006

Original article is available at http://www.emis.de/journals/SIGMA/2006/Paper034/

\section{Submitted by Anatoly Klimyk}

\begin{abstract}
The dual discrete $q$-ultraspherical polynomials $D_{n}^{(s)}(\mu(x ; s) \mid q)$ correspond to indeterminate moment problem and, therefore, have one-parameter family of extremal orthogonality relations. It is shown that special cases of dual discrete $q$-ultraspherical polynomials $D_{n}^{(s)}(\mu(x ; s) \mid q)$, when $s=q^{-1}$ and $s=q$, are directly connected with $q^{-1}$-Hermite polynomials. These connections are given in an explicit form. Using these relations, all extremal orthogonality relations for these special cases of polynomials $D_{n}^{(s)}(\mu(x ; s) \mid q)$ are found.
\end{abstract}

Key words: $q$-orthogonal polynomials; dual discrete $q$-ultraspherical polynomials; $q^{-1}$-Hermite polynomials; orthogonality relation

2000 Mathematics Subject Classification: 33D45; 81Q99

\section{Introduction}

Quantum groups and $q$-deformed variants of the quantum harmonic oscillator were introduced approximately two decades ago. Representations of quantum groups and realizations of $q$ oscillators are closely related to basic hypergeometric functions and $q$-orthogonal polynomials. Instead of Jacobi, Gegenbauer (ultraspherical), Hermite polynomials, in the theory of quantum groups and under studying $q$-oscillators we deal with different kinds of $q$-Jacobi polynomials, of $q$-ultraspherical polynomials and of $q$-Hermite polynomials.

It is known that $q$-orthogonal polynomials are more complicated than classical orthogonal polynomials. It is interesting that main development of the theory of $q$-orthogonal polynomials in fact coincides in time with appearing of quantum groups (see, for example, [1] and [2]). Due to great importance for contemporary mathematical and theoretical physics the theory of $q$ orthogonal polynomials is under extensive development. A great attention is paid to those of them, which are directly related to representations of quantum groups and to realizations of $q$-oscillators.

In this paper we deal with the so-called $q^{-1}$-Hermite polynomials (which are closely related to the Biedenharn-Macfarlane oscillator; see [3]) and with the dual discrete $q$-ultraspherical polynomials. The $q^{-1}$-Hermite orthogonal polynomials were discovered by Askey [4] and the discrete $q$-ultraspherical polynomials and their duals were introduced in [5].

The $q^{-1}$-Hermite orthogonal polynomials correspond to indeterminate moment problem. This means that they have a one-parameter family of orthogonality relations (these orthogonalities 
are important for studying the Biedenharn-Macfarlane oscillator when $q>1$; see [3]). These orthogonality relations were explicitly derived in [6]. It was shown in [5] that dual $q$-ultraspherical polynomials also correspond to indeterminate moment problem and, therefore, there exists a oneparameter family of orthogonality relations for them. In [5], there were derived only two orthogonality relations for these polynomials. Other orthogonality relations for them are not known. As in the case of usual ultraspherical (Gegenbauer) polynomials, these orthogonality relations are very important.

Let us remark that finding extremal orthogonality relations for orthogonal polynomials, which correspond to indeterminate moment problems, is a very difficult task. In fact, at present only one example of such measures is known; that is extremal orthogonality relations for the $q^{-1}$. Hermite polynomials.

In this paper we derive explicit formulas that connect the dual $q$-ultraspherical polynomials $D_{n}^{(s)}(\mu(x ; s) \mid q)$, when $s=q^{-1}$ and $s=q$, with the $q^{-1}$-Hermite polynomials, and this gives a possibility to obtain all extremal measures for these particular types of the the dual $q$-ultraspherical polynomials.

Note that the dual $q$-ultraspherical polynomials $D_{n}^{(s)}(\mu(x ; s) \mid q)$ are related to the discrete series representations of the quantum Drinfeld-Jimbo algebra $U_{q}\left(\mathrm{su}_{1,1}\right)$; see [7].

Below we use (without additional explanation) notations of the theory of $q$-special functions (see [8]). In particular, the shifted $q$-factorials $(a ; q)_{n}$ are defined as

$$
(a ; q)_{n}=(1-a)(1-a q)\left(1-a q^{2}\right) \cdots\left(1-a q^{n-1}\right) .
$$

The basic hypergeometric functions are given by the formula

$$
{ }_{r} \phi_{r-1}\left(a_{1}, a_{2}, \ldots, a_{r} ; b_{1}, b_{2}, \ldots, b_{s} ; q, x\right)=\sum_{n=0}^{\infty} \frac{\left(a_{1} ; q\right)_{n}\left(a_{2} ; q\right)_{n} \ldots\left(a_{r} ; q\right)_{n}}{\left(b_{1} ; q\right)_{n}\left(b_{2} ; q\right)_{n} \ldots\left(b_{r-1} ; q\right)_{n}} \frac{x^{n}}{(q ; q)_{n}} .
$$

\section{Dual discrete $q$-ultraspherical polynomials and $q^{-1}$-Hermite polynomials}

The discrete $q$-ultraspherical polynomials $C_{n}^{(s)}(x ; q)$ are the polynomials

$$
C_{n}^{(s)}(x ; q)={ }_{3} \phi_{2}\left(q^{-n},-s q^{n+1}, x ; \sqrt{s} q,-\sqrt{s} q ; q, q\right) .
$$

These polynomials are orthogonal on a countable set of points (see formula (4) in [5]).

In [9], the procedure of creation of dual sets of polynomials, orthogonal on a discrete set of points, is being developed. The polynomials $D_{n}^{(s)}(\mu(x ; s) ; q)$ dual to the discrete $q$-ultraspherical polynomials $C_{n}^{(s)}(x ; q)$ are of particular case of the dual big $J$-Jacobi polynomials (see [10]). They are given by the formula

$$
D_{n}^{(s)}(\mu(x ; s) ; q)={ }_{3} \phi_{2}\left(q^{-x}, s q^{x+1}, q^{-n} ; \sqrt{s} q,-\sqrt{s} q ; q,-q^{n+1}\right),
$$

where $\mu(x ; s)=q^{-x}+s q^{x+1}$. They are polynomials in $\mu(x ; s)$. These polynomials satisfy the three-term recurrence relation

$$
\begin{aligned}
\left(q^{-x}\right. & \left.+s q^{x+1}\right) D_{n}^{(s)}(\mu(x ; s) \mid q)=-q^{-2 n-1}\left(1-s q^{2 n+2}\right) D_{n+1}^{(s)}(\mu(x ; s) \mid q) \\
& +q^{-2 n-1}(1+q) D_{n}^{(s)}(\mu(x ; s) \mid q)-q^{-2 n}\left(1-q^{2 n}\right) D_{n-1}^{(s)}(\mu(x ; s) \mid q) .
\end{aligned}
$$

They correspond to indeterminate moment problem and it means that they satisfy a oneparameter family of orthogonality relations. Only two orthogonality relations were found in [5]:

$$
\sum_{k=0}^{\infty} \frac{\left(1-s q^{4 k+1}\right)(s q ; q)_{2 k}}{(1-s q)(q ; q)_{2 k}} q^{k(2 k-1)} D_{n}^{(s)}(\mu(2 k) \mid q) D_{n^{\prime}}^{(s)}(\mu(2 k) \mid q)
$$




$$
\begin{aligned}
&=\frac{\left(s q^{3} ; q^{2}\right)_{\infty}}{\left(q ; q^{2}\right)_{\infty}} \frac{\left(q^{2} ; q^{2}\right)_{n} q^{-n}}{\left(s q^{2} ; q^{2}\right)_{n}} \delta_{n n^{\prime}}, \\
& \sum_{k=0}^{\infty} \frac{\left(1-s q^{4 k+3}\right)(s q ; q)_{2 k+1}}{(1-s q)(q ; q)_{2 k+1}} q^{k(2 k+1)} D_{n}^{(s)}(\mu(2 k+1) \mid q) D_{n^{\prime}}^{(s)}(\mu(2 k+1) \mid q) \\
&=\frac{\left(s q^{3} ; q^{2}\right)_{\infty}}{\left(q ; q^{2}\right)_{\infty}} \frac{\left(q^{2} ; q^{2}\right)_{n} q^{-n}}{\left(s q^{2} ; q^{2}\right)_{n}} \delta_{n n^{\prime}},
\end{aligned}
$$

where $\mu(2 k) \equiv \mu(2 k ; s), \mu(2 k+1) \equiv \mu(2 k+1 ; s)$ and $0<s<q^{-2}$.

We shall need also $q^{-1}$-Hermite polynomials $h_{n}(x \mid q)$, which are given as

$$
h_{n}(\sinh \varphi \mid q)=\sum_{k=0}^{n} \frac{(-1)^{k} q^{k(k-n)}(q ; q)_{n}}{(q ; q)_{k}(q ; q)_{n-k}} e^{\varphi(n-2 k)} \text {. }
$$

They are defined as $h_{n}(x \mid q)=\mathrm{i}^{-n} H_{n}\left(\mathrm{i} x \mid q^{-1}\right)$, where $H_{n}(y \mid q)$ are well-known continuous $q$ Hermite polynomials. The polynomials $h_{n}(x \mid q)$ satisfy the following recurrence relation

$$
\left.h_{n+1}(x \mid q)+q^{-n}\left(1-q^{n}\right) h_{n-1}(x \mid q)\right)=2 x h_{n}(x \mid q) \text {. }
$$

It follows from this relation that the polynomials $h_{2 k}(x \mid q)$ are even:

$$
h_{2 k}(-x \mid q)=h_{2 k}(x \mid q)
$$

and the polynomials $h_{2 k+1}(x \mid q)$ have the form

$$
h_{2 k+1}(x \mid q)=x \tilde{h}_{2 k}(x \mid q) \text {, }
$$

where $\tilde{h}_{2 k}(x \mid q)$ are even polynomials: $\tilde{h}_{2 k}(-x \mid q)=\tilde{h}_{2 k}(x \mid q)$.

The $q^{-1}$-Hermite polynomials $h_{n}(x \mid q)$ correspond to indeterminate moment problem and, therefore, have one-parameter family of extremal orthogonality measures, which were given in [6]. They are given by the parameter $a, q \leq a<1$, and have the form

$$
\sum_{m=-\infty}^{\infty} \frac{a^{4 m} q^{m(2 m-1)}\left(1+a^{2} q^{2 m}\right)}{\left(-a^{2} ; q\right)_{\infty}\left(-q / a^{2} ; q\right)_{\infty}(q ; q)_{\infty}} h_{n}\left(x_{m} \mid q\right) h_{n^{\prime}}\left(x_{m} \mid q\right)=q^{-n(n+1) / 2}(q ; q)_{n} \delta_{n n^{\prime}}
$$

where

$$
x_{m}=\frac{1}{2}\left(a^{-1} q^{-m}-a q^{m}\right) .
$$

The relations (5) were proved in [6] only for $a$ from the interval $q<a<1$. It is only said there that the relation (5) is true also for $a=q$. Below we shall give a proof of (5) for $a=q$.

Proposition. The following expression for $q^{-1}$-Hermite polynomials $h_{n}(x \mid q)$ hold:

$$
\begin{aligned}
& h_{2 k}(\sinh \varphi \mid q)=(-1)^{k} q^{-k^{2}}\left(q ; q^{2}\right)_{k} D_{k}^{\left(q^{-1}\right)}\left(e^{2 \varphi}+e^{-2 \varphi} \mid q\right), \\
& h_{2 k+1}(\sinh \varphi \mid q)=(-1)^{k} q^{-k(k+1)}\left(q^{3} ; q^{2}\right)_{k}(2 \sinh \varphi) D_{k}^{(q)}\left(q e^{2 \varphi}+q e^{-2 \varphi} \mid q\right),
\end{aligned}
$$

where $k$ are nonnegative integers and $D_{k}^{(s)}(\mu(m ; s) \mid q)$ are dual discrete $q$-ultraspherical polynomials. 
Proof. We take the recurrence relation (4) for $n=2 k$ :

$$
\left.2 x h_{2 k}(x \mid q)=h_{2 k+1}(x \mid q)+q^{-2 k}\left(1-q^{2 k}\right) h_{2 k-1}(x \mid q)\right)
$$

and substitute there the expressions for $h_{2 k+1}(x \mid q)$ and $h_{2 k-1}(x \mid q)$, obtained from (4). As a result, we get the relation

$$
\begin{aligned}
(2 x)^{2} h_{2 k}(x \mid q)= & h_{2 k+2}(x \mid q)+\left[q^{-2 k}\left(1+q^{-1}\right)-2\right] h_{2 k}(x \mid q) \\
& +q^{-4 k+1}\left(1-q^{2 k}\right)\left(1-q^{2 k-1}\right) h_{2 k-2}(x \mid q)
\end{aligned}
$$

which can be written in the form

$$
\begin{aligned}
\left(e^{2 \varphi}+e^{-2 \varphi}\right) h_{2 k}(\sinh \varphi \mid q)= & h_{2 k+2}(\sinh \varphi \mid q)+q^{-2 k}\left(1+q^{-1}\right) h_{2 k}(\sinh \varphi \mid q) \\
& +q^{-4 k+1}\left(1-q^{2 k}\right)\left(1-q^{2 k-1}\right) h_{2 k-2}(\sinh \varphi \mid q) .
\end{aligned}
$$

Now we take the recurrence relation (1) for $s=q^{-1}$ :

$$
\begin{aligned}
y D_{n}^{\left(q^{-1}\right)}(y \mid q)= & -q^{-2 n-1}\left(1-q^{2 n+1}\right) D_{n+1}^{\left(q^{-1}\right)}(y \mid q)+q^{-2 n-1}(1+q) D_{n}^{\left(q^{-1}\right)}(y \mid q) \\
& -q^{-2 n}\left(1-q^{2 n}\right) D_{n-1}^{\left(q^{-1}\right)}(y \mid q)
\end{aligned}
$$

and substitute there the expression

$$
D_{n}^{\left(q^{-1}\right)}(y \mid q)=(-1)^{n} q^{n^{2}}\left(q ; q^{2}\right)_{n}^{-1} \tilde{D}_{n}^{\left(q^{-1}\right)}(y \mid q)
$$

(compare it with the first relation in the proposition), where $\tilde{D}_{n}^{(s)}(y \mid q)$ are polynomials multiple to the polynomials $D_{n}^{(s)}(y \mid q)$. As a result, we obtain the relation

$$
\begin{aligned}
y \tilde{D}_{n}^{\left(q^{-1}\right)}(y \mid q)= & \tilde{D}_{n+1}^{\left(q^{-1}\right)}(y \mid q)+q^{-2 n-1}(1+q) \tilde{D}_{n}^{\left(q^{-1}\right)}(y \mid q) \\
& +q^{-4 n+1}\left(1-q^{2 n}\right)\left(1-q^{2 n-1}\right) \tilde{D}_{n-1}^{\left(q^{-1}\right)}(y \mid q) .
\end{aligned}
$$

Setting here $y=e^{2 \varphi}+e^{-2 \varphi}$ and then comparing the relations (8) and (9) we get

$$
h_{2 k}(\sinh \varphi \mid q)=c \tilde{D}_{k}^{\left(q^{-1}\right)}\left(e^{2 \varphi}+e^{-2 \varphi} \mid q\right),
$$

where $c$ is a constant. In order to find this constant we put $k=0$ in (10). Since $h_{0}(x \mid q)=1$ and $\tilde{D}_{0}^{\left(q^{-1}\right)}(y \mid q)=1$, we obtain that $c=1$ and the first relation of the proposition is true.

The relation (7) is proved similarly. We take the recurrence relation (4) for $n=2 k+1$ :

$$
2 x h_{2 k+1}(x \mid q)=h_{2 k+2}(x \mid q)+q^{-2 k-1}\left(1-q^{2 k+1}\right) h_{2 k}(x \mid q)
$$

and substitute there the expressions for $h_{2 k+2}(x \mid q)$ and $h_{2 k}(x \mid q)$, obtained from (4). As a result, we get the relation which can be written in the form

$$
\begin{aligned}
& \left(q e^{2 \varphi}+q e^{-2 \varphi}\right) h_{2 k+}(\sinh \varphi \mid q)=q h_{2 k+3}(\sinh \varphi \mid q) \\
& \quad+q^{-2 k}\left(1+q^{-1}\right) h_{2 k+1}(\sinh \varphi \mid q)+q^{-4 k}\left(1-q^{2 k}\right)\left(1-q^{2 k+1}\right) h_{2 k}(\sinh \varphi \mid q) .
\end{aligned}
$$

Now we take the recurrence relation (1) for $s=q$ :

$$
\begin{aligned}
y D_{n}^{(q)}(y \mid q)= & -q^{-2 n-1}\left(1-q^{2 n+3}\right) D_{n+1}^{(q)}(x \mid q)+q^{-2 n-1}(1+q) D_{n}^{(q)}(y \mid q) \\
& -q^{-2 n}\left(1-q^{2 n}\right) D_{n-1}^{(q)}(y \mid q)
\end{aligned}
$$


and substitute there the expression

$$
D_{n}^{(q)}(y \mid q)=(-1)^{n} q^{n(+1)}\left(q^{3} ; q^{2}\right)_{n}^{-1} \tilde{D}_{n}^{(q)}(y \mid q)
$$

(compare with the second relation in the proposition). As a result, we obtain the relation

$$
\begin{aligned}
y \tilde{D}_{n}^{\left(q^{-1}\right)}(y \mid q)= & q \tilde{D}_{n+1}^{\left(q^{-1}\right)}(y \mid q)+q^{-2 n-1}(1+q) \tilde{D}_{n}^{\left(q^{-1}\right)}(y \mid q) \\
& +q^{-4 n}\left(1-q^{2 n}\right)\left(1-q^{2 n+1}\right) \tilde{D}_{n-1}^{\left(q^{-1}\right)}(y \mid q) .
\end{aligned}
$$

Setting here $y=q e^{2 \varphi}+q e^{-2 \varphi}$ and then comparing the relations (11) and (12) we get

$$
h_{2 k+1}(\sinh \varphi \mid q)=c \tilde{D}_{k}^{(q)}\left(e^{2 \varphi}+e^{-2 \varphi} \mid q\right),
$$

where $c$ is a constant. Putting $k=0$ in (13), we find that $c=1$ and the second relation of the proposition is true. The proposition is proved.

\section{Orthogonality relations for $h_{n}(x \mid q)$ when $a=q$}

The aim of this section is to show that the orthogonality relations (2) and (3) are equivalent to to the orthogonality relation (5) for $a=q$. Thus, we give a proof of the orthogonality relation (5) for $a=q$, starting from the orthogonality relations (2) and (3).

It follows from relation (2) that the dual discrete $q$-ultraspherical polynomials $D_{n}^{\left(q^{-1}\right)}(x \mid q)$ satisfy the orthogonality relation

$$
\sum_{m=0}^{\infty}\left(1+q^{2 m}\right) q^{m(2 m-1)} D_{n}^{\left(q^{-1}\right)}\left(y_{m} \mid q\right) D_{n^{\prime}}^{\left(q^{-1}\right)}\left(y_{m} \mid q\right)=\frac{\left(q^{2} ; q^{2}\right)_{\infty}}{\left(q ; q^{2}\right)_{\infty}} \frac{\left(q^{2} ; q^{2}\right)_{n} q^{-n}}{\left(q ; q^{2}\right)_{n}} \delta_{n n^{\prime}},
$$

where $y_{m}=q^{2 m}+q^{-2 m}$. By using Proposition, we express $D_{n}^{\left(q^{-1}\right)}\left(x_{m} \mid q\right)$ in terms of $h_{2 n}\left(\frac{1}{2}\left(q^{-m}-\right.\right.$ $\left.q^{m}\right) \mid q$ ) and substitute into (14). As a result, we obtain

$$
\sum_{m=0}^{\infty}\left(1+q^{2 m}\right) q^{m(2 m-1)} h_{2 n}\left(x_{m} \mid q\right) h_{2 n^{\prime}}\left(x_{m} \mid q\right)=\frac{\left(q^{2} ; q^{2}\right)_{\infty}}{\left(q ; q^{2}\right)_{\infty}} q^{-n(2 n+1)}(q ; q)_{2 n} \delta_{n n^{\prime}},
$$

where $x_{m}=\frac{1}{2}\left(q^{-m}-q^{m}\right)$. Note that

$$
\begin{aligned}
\frac{\left(q^{2} ; q^{2}\right)_{\infty}}{\left(q ; q^{2}\right)_{\infty}} & =(-q ; q)_{\infty}^{2}(q ; q)_{\infty}=\frac{1}{2}(-1 ; q)_{\infty}(-q ; q)_{\infty}(q ; q)_{\infty} \\
& =2 q^{-1}\left(-q^{2} ; q\right)_{\infty}\left(-q^{-1} ; q\right)_{\infty}(q ; q)_{\infty} .
\end{aligned}
$$

The expression $\left(1+q^{2 m}\right) q^{m(2 m-1)}$ on the right hand side of (15) does not change at replacing $m$ by $-m$. Since $h_{2 n}(x \mid q)$ is an even function, then due to (16) the relation (15) can be written as

$$
\sum_{m=-\infty}^{\infty} \frac{\left(1+q^{2 m}\right) q^{-1} q^{m(2 m-1)}}{\left(-q^{2} ; q\right)_{\infty}\left(-q^{-1} ; q\right)_{\infty}(q ; q)_{\infty}} h_{2 n}\left(x_{m} \mid q\right) h_{2 n^{\prime}}\left(x_{m} \mid q\right)=q^{-n(2 n+1)}(q ; q)_{2 n} \delta_{n n^{\prime}} .
$$

This relation is equivalent to the orthogonality relation (5) at $a=q$ if $n$ and $n^{\prime}$ are replaced there by $2 n$ and $2 n^{\prime}$, and $m$ by $-m-1$, respectively.

Now we use the relation (3) for the dual discrete $q$-ultraspherical polynomials $D_{n}^{(q)}(x \mid q)$. Then after shifting $k$ to $k-1$ we have

$$
\sum_{m=1}^{\infty}\left(1+q^{2 m}\right)\left(1-q^{2 m}\right)^{2} q^{(m-1)(2 m-1)} D_{n}^{(q)}\left(y_{m} \mid q\right) D_{n^{\prime}}^{(q)}\left(y_{m} \mid q\right)
$$




$$
=\frac{\left(q^{2} ; q^{2}\right)_{\infty}}{\left(q ; q^{2}\right)_{\infty}} \frac{(1-q)^{2}\left(q^{2} ; q^{2}\right)_{n} q^{-n}}{\left(1-q^{2 n+1}\right)\left(q ; q^{2}\right)_{n}} \delta_{n n^{\prime}}
$$

where $y_{m}=q^{2 m+1}+q^{-2 m+1}$. By using the above Proposition, we express $D_{n}^{(q)}\left(y_{m} \mid q\right)$ in terms of $h_{2 n+1}\left(\frac{1}{2}\left(q^{m}-q^{-m}\right) \mid q\right)$ and substitute into (18). We obtain

$$
\begin{gathered}
\sum_{m=1}^{\infty}\left(1+q^{2 m}\right) q^{m(2 m-1)} h_{2 n+1}\left(x_{m} \mid q\right) h_{2 n^{\prime}+1}\left(x_{m} \mid q\right) \\
=\frac{\left(q^{2} ; q^{2}\right)_{\infty}}{\left(q ; q^{2}\right)_{\infty}} q^{-(n+1)(2 n+1)}(q ; q)_{2 n+1} \delta_{n n^{\prime}}
\end{gathered}
$$

where, as before, $x_{m}=\frac{1}{2}\left(q^{-m}-q^{m}\right)$. Since $h_{2 n+1}(0 \mid q)=0$, then the summation $\sum_{m=1}^{\infty}$ here can be replaced by $\sum_{m=0}^{\infty}$, and then by $\frac{1}{2} \sum_{m=-\infty}^{\infty}$ (since $h_{2 n+1}(x \mid q)$ is an odd function). As in the case of (15), it is easy to show that the last relation is equivalent to the relation (5) if we replace $m$ by $m-1$, and $n$ and $n^{\prime}$ by $2 n+1$ and $2 n^{\prime}+1$, respectively.

Since the polynomials $h_{2 n}\left(x_{m} \mid q\right)$ are even and the polynomials $h_{2 n+1}\left(x_{m} \mid q\right)$ are odd, then

$$
\begin{aligned}
\sum_{m=0}^{\infty}( & \left.+q^{2 m}\right) q^{m(2 m-1)} h_{2 n}\left(x_{m} \mid q\right) h_{2 n^{\prime}+1}\left(x_{m} \mid q\right) \\
& =-\sum_{m=-\infty}^{0}\left(1+q^{2 m}\right) q^{m(2 m-1)} h_{2 n}\left(x_{m} \mid q\right) h_{2 n^{\prime}+1}\left(x_{m} \mid q\right) .
\end{aligned}
$$

Therefore,

$$
\sum_{m=-\infty}^{\infty}\left(1+q^{2 m}\right) q^{m(2 m-1)} h_{2 n}\left(x_{m} \mid q\right) h_{2 n^{\prime}+1}\left(x_{m} \mid q\right)=0 .
$$

This gives the mutual orthogonality of the polynomials $h_{2 n}\left(x_{m} \mid q\right), n=0,1,2, \ldots$, to the polynomials $h_{2 n+1}\left(x_{m} \mid q\right), n=0,1,2, \ldots$. Thus, we proved the relation (5) for $a=q$.

\section{Orthogonality relations for the polynomials $D_{n}^{\left(q^{-1}\right)}$ and $D_{n}^{(q)}$}

In this section we use the orthogonality relations for the $q^{-1}$-Hermite polynomials in order to find orthogonality relations for the dual discrete $q$-ultraspherical polynomials $D_{n}^{\left(q^{-1}\right)}$ and $D_{n}^{(q)}$. We substitute the expression (6) for $h_{2 k}(\sinh \varphi \mid q)$ into the orthogonality relation (5). After some transformations we receive the orthogonality relations for $D_{n}^{\left(q^{-1}\right)}$ :

$$
\begin{aligned}
& \sum_{m=-\infty}^{\infty} a^{4 m+1} q^{2 m^{2}}\left(a^{-1} q^{-m}+a q^{m}\right) D_{k}^{\left(q^{-1}\right)}\left(y_{m} \mid q\right) D_{k}^{\left(q^{-1}\right)}\left(y_{m} \mid q\right) \\
& =\frac{\left(-a^{2} ; q\right)_{\infty}(-q / a ; q)_{\infty}(q ; q)_{\infty}}{q^{k}(q ; q)_{2 k}^{-1}\left(q ; q^{2}\right)_{k}^{2}} \delta_{k k^{\prime}},
\end{aligned}
$$

where

$$
y_{m}=a^{-2} q^{-2 m}+a^{2} q^{2 m} .
$$

These relations are parametrized by $a, q \leq a<1$. For different values of $a$ the corresponding orthogonality relations are not equivalent. 
Now we substitute into (5) the relation (7) for $h_{2 k+1}(\sinh \varphi \mid q)$. After some transformation we obtain

$$
\begin{aligned}
& \sum_{m=-\infty}^{\infty} a^{4 m} q^{m(2 m-1)}\left(a^{-2} q^{-2 m}-a^{2} q^{2 m}\right) D_{k}^{(q)}\left(y_{m} \mid q\right) D_{k}^{(q)}\left(y_{m} \mid q\right) \\
& =\frac{\left(-a^{2} ; q\right)_{\infty}(-q / a ; q)_{\infty}(q ; q)_{\infty}}{q^{k+1}(q ; q)_{2 k+1}^{-1}\left(q^{3} ; q^{2}\right)_{k}^{2}} \delta_{k k^{\prime}}
\end{aligned}
$$

where

$$
y_{m}=\left(a^{-2} q^{-2 m}+a^{2} q^{2 m}\right) q .
$$

These relations are also parametrized by $a, q \leq a<1$. Orthogonality relations, corresponding to different values of $a$, are not equivalent.

The orthogonality relations (5) for the $q^{-1}$-Hermite polynomials are extremal, that is, the set of polynomials $h_{n}\left(x_{m} \mid q\right), n=0,1,2, \ldots$, is complete in the corresponding Hilbert space (see [11]). Therefore, the orthogonality relations (21) and (22) for the polynomials $D_{k}^{\left(q^{-1}\right)}$ and $D_{k}^{(q)}$, respectively, are extremal. Moreover, since the set of orthogonality relations (5) is a complete set of extremal orthogonality relations for the $q^{-1}$-Hermite polynomials, then the set of relations (21) is a complete set of extremal orthogonality relations for the polynomials $D_{k}^{\left(q^{-1}\right)}$ and the set of relations $(22)$ is a complete set of extremal orthogonality relations for the polynomials $D_{k}^{(q)}$.

\section{Concluding remarks}

The dual discrete $q$-ultraspherical polynomials $D_{n}^{(s)}(\mu(x ; s) \mid q)$ correspond to the indeterminate moment problem, that is, there are infinitely many (one-parameter family) of orthogonality relations for them. We have found these orthogonality relations for the cases when $s=q$ and $s=q^{-1}$. Namely, we have reduced these cases to the $q^{-1}$-Hermite polynomials for which orthogonality relations are known. It leaves unsolved the problem of finding orthogonality relations for other cases. We believe that the orthogonality relations (21) and (22) will help in finding orthogonality relations for these other cases.

There exists another class of dual discrete $q$-ultraspherical polynomials (they are denoted as $\tilde{D}_{n}^{(s)}(\mu(x ;-s) \mid q)$; see [5, Section 1]). They also correspond to the indeterminate moment problem. It is interesting to find orthogonality relations for them. However, they cannot be reduced to the $q^{-1}$-Hermite polynomials. It is shown in [5] that they are reduced to BergIsmail polynomials. This connection gives an infinite number of orthogonality relations for them. However, they are not extremal orthogonality relations. So, the problem of finding these orthogonality relations are unsolved.

It is well-known (see [3]) that $q^{-1}$-Hermite polynomials are closely related to the BiedenharnMacfarlane $q$-oscillator. Thus, the polynomials, considered in this paper, is also related to this $q$-oscillator. Then the following question appears: Are the polynomials $\tilde{D}_{n}^{(s)}(\mu(x ;-s) \mid q)$ related to the $q$-oscillator? Probably, they correspond to some simple deformation of the $q$-oscillator. 
[1] Askey R., Wilson J., Some basic hypergeometric polynomials that generalize Jacobi polynomials, Memoirs Amer. Math. Soc., 1985, V.319, 1-115.

[2] Askey R., Wilson J., A set of orthogonal polynomials that generalize the Racah coefficients or $6-j$ symbols, SIAM J. Math. Anal., 1979, V.10, 1008-1016.

[3] Klimyk A., Spectra of observables in the $q$-oscillator and $q$-analogue of the Fourier transform, SIGMA, 2005, V.1, Paper 008, 17 pages, math-ph/0508032.

[4] Askey R., Continuous $q$-Hermite polynomials when $q>1$, in $q$-Series and Partitions, Editor D. Stanton, Berlin, Springer, 1998, 151-158.

[5] Atakishiyev N.M., Klimyk A.U., On discrete q-ultraspherical polynomials and their duals, J. Math. Anal. Appl., 2005, V.306, 637-645, math.CA/0403159.

[6] Ismail M.E.R., Masson D.R., q-Hermite polynomials, biorthogonal functions, and $q$-beta integrals, Trans. Amer. Math. Soc., 1994, V.346, 63-116.

[7] Groza V., Representations of the quantum algebra $s u_{q}(1,1)$ and discrete $q$-ultraspherical polynomials, SIGMA, 2005, V.1, Paper 016, 7 pages, math.QA/0511632.

[8] Gasper G., Rahman M., Basic hypergeometric functions, Cambridge, Cambridge University Press, 1990.

[9] Atakishiyev N.M., Klimyk A.U., Duality of $q$-polynomials, orthogonal on countable sets of points, Elect. Trans. Numer. Anal., to appear, math.CA/0411249.

[10] Atakishiyev N.M., Klimyk A.U., On $q$-orthogonal polynomials, dual to little and big $q$-Jacobi polynomials, J. Math. Anal. Appl., 2004, V.294, 246-257, math.CA/0307250.

[11] Shohat J., Tamarkin J.D., The problem of moments, Providence, R.I., American Mathematical Society, 1943. 